\title{
The Influence of COVID-19 on the Stability of Patients with Neovascular Age-Related Macular Degeneration with Different Treatment Regimens
}

\author{
Bo Jiang · Lin Gao · Su Dong · Qingxue Hou · Minghao Sun · \\ Jingjie Zhang $\cdot$ Haotian Yu $\cdot$ Zhongyu Zhang $\cdot$ Dawei Sun (D)
}

Received: September 29, 2021 / Accepted: November 9, 2021 / Published online: November 24, 2021

(C) The Author(s) 2021, corrected publication 2022

\begin{abstract}
Introduction: To explore the impact of coronavirus disease 2019 (COVID-19) on the stability of patients with neovascular age-related macular degeneration (nAMD) receiving the treat and extend (T\&E) or the pro re nata (PRN) treatment regimen and to identify indicators that may predict the disease stability of nAMD.

Methods: This is a retrospective study of patients with nAMD treated at the Second Affiliated Hospital of Harbin Medical University whose treatment schedule was interrupted at least once between 1 February and 31 May 2020. The demographic and clinical characteristics, including the best corrected visual acuity (BCVA), optical coherence tomography (OCT) features, subfoveal choroidal thickness (SFCT), interval between the last injection and the beginning of the pandemic, and the number of anti-vascular endothelial growth factor (VEGF) injections, were analyzed.
\end{abstract}

Results: A total of 209 stable patients with nAMD (122 eyes received the T\&E regimen; 87

Bo Jiang and Lin Gao contributed equally to this work.

B. Jiang $\cdot$ L. Gao $\cdot$ S. Dong $\cdot$ Q. Hou $\cdot$ M. Sun ·

J. Zhang · H. Yu . Z. Zhang · D. Sun (凶)

Ophthalmology Department, The Second Affiliated

Hospital of Harbin Medical University, 246 Xuefu

Road, Nangang District, Harbin 150001,

Heilongjiang, China

e-mail: sun.dawei@hotmail.com eyes received the PRN regimen) were identified. Compared to those who received the PRN regimen, the patients who received the T\&E regimen were more stable during the first visit after COVID-19 (53.3\% vs. $33.3 \%, P=0.004)$, the BCVA was significantly better ( 58.5 letters vs. 56 letters, $P=0.006)$, and the CRT fluctuated only slightly $(15 \mu \mathrm{m}$ vs. $35 \mu \mathrm{m}, P=0.001)$. Furthermore, a multivariate logistic regression analysis showed that stable patients with nAMD with type 1 choroidal neovascularization (CNV) (OR 2.493 [95\% CI 1.179-5.272], compared with type $2 \mathrm{CNV} ; \quad P=0.017$; OR $2.912 \quad$ [95\% CI 1.133-7.485], compared with retinal angiomatous proliferation; $P=0.026$ ) or with pigment epithelial detachment (PED) were more likely to remain stable when treatment was interrupted (OR 0.392 [95\% CI 0.181-0.852], compared with no PED; $P=0.018$ ).

Conclusion: Compared to patients who received the PRN treatment regimen, stable patients with nAMD who received the T\&E treatment regimen could better maintain stability when the treatments were suddenly interrupted by the COVID-19 pandemic. In addition, patients with type $1 \mathrm{CNV}$ or patients with PED were more likely to remain stable. At present, the COVID-19 pandemic is becoming increasingly normalized, and the T\&E regimen can become a more advanced treatment option for patients undergoing therapy. 
Keywords: COVID-19; nAMD; PRN regimen; Stability; T\&E regimen

\section{Key Summary Points}

Why carry out this study?

The outbreak of COVID-19 caused the interruption of anti-vascular endothelial growth factor (VEGF) treatment in patients with Neovascular age-related macular degeneration (nAMD), even resulting in the deterioration of previously stable patients. Therefore, we need to explore a regimen that is more beneficial to maintain the stability of patients.

What was learned from the study?

The treat and extend (T\&E) regimen can better maintain the stability of the treatment effect seen in patients with nAMD. After the COVID-19 interruption, the patients' visual acuities decreased less, the patients' lesions reactivated less, and the treatment was more regular. The COVID-19 pandemic is becoming increasingly normalized, and the T\&E regimen should be a more advanced treatment option in the clinic.

\section{INTRODUCTION}

Neovascular age-related macular degeneration (nAMD) is a major cause of visual impairment in elderly people worldwide [1-3]. Anti-vascular endothelial growth factor (VEGF) therapy has been used as the gold standard treatment method for nAMD to prevent disease progression and to provide functional and anatomical improvement [4-7]. However, timely administration of the intravitreal injections at appropriate intervals and regular follow-up of the patients are crucial for the success of the treatment. Most randomized controlled trials use a fixed dosing of anti-VEGF agents, which requires monthly or bimonthly injections $[8,9]$. Although the fixed-dosing regimen has great potential to improve and maintain vision, the frequency of treatment requires a high level of patient adherence. Moreover, increasing the treatment burden on doctors and patients might cause more difficulty with their compliance. Additionally, it brings high economic costs to patients, which could be an obstacle in clinical practice [10]. The pro re nata (PRN) regimen is a treatment regimen widely used in the clinic at present. The frequency of the injections during treatment is determined on the basis of the activity of the disease when the patients have symptoms of visual impairment or on the basis of the activity indicators during follow-up [11]. Although the PRN regimen reduces the frequency of treatment, patients still need to be monitored every month, and the compliance requirements of the patients are still high.

The treat and extend (T\&E) protocol is a personalized active treatment regimen [12]. It mandates that at least three consecutive monthly injections continue until the patient develops a stable state that is determined when the retina is dry and when there are no other signs of disease activity, after which the interval between visits and the injections can be extended or shortened on the basis of the clinician's assessment of the patient's disease activity [13]. Compared with the monthly and PRN regimens, the T\&E regimen can significantly reduce the number of follow-up visits and injections and can ease the treatment burden of patients. The T\&E regimen has been proven to achieve a comparable efficacy to the traditional fixed dosing and the PRN regimens [14-16].

Coronavirus disease 2019 (COVID-19) is an infectious disease that has rapidly spread worldwide and poses a serious threat to public health and safety $[17,18]$. As a result of the sudden epidemic, many medical institutions had to stop clinical work, and the routine treatment of many patients was interrupted, including those receiving anti-VEGF treatment. 


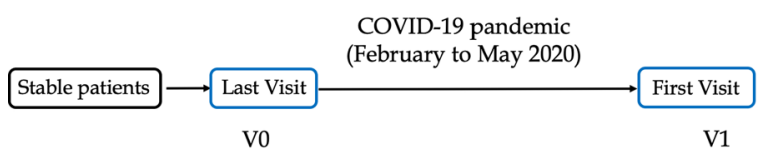

Fig. 1 Follow-up of patients with nAMD before and after COVID-19 pandemic. The last pre-epidemic visit was recorded as $\mathrm{V} 0$, the $\mathrm{T} \& \mathrm{E}$ regime patients received the last injection at V0, and the PRN regime patients assessed whether to receive injection according to stable criteria at V0. The first follow-up after the pandemic was recorded as $\mathrm{V} 1$, and the patients were assessed as stable or not at V1 on the basis of the evaluation criteria of stable nAMD

Untreated disease or delayed treatment with anti-VEGF will result in patients with severe visual impairment during the initial diagnosis of nAMD, and for patients with nAMD who have already begun to receive anti-VEGF treatment, a sudden interruption or delay in treatment secondary to interruptions by the COVID19 pandemic can also result in decreased visual acuity and a recurrence of the lesions $[19,20]$. However, it is not clear whether patients with nAMD who have reached a stable state after a course of anti-VEGF treatment will be affected by the COVID-19 pandemic. It is also unclear whether patients with nAMD who achieve a stable state with different treatment regimens can maintain the stable state if treatment is stopped or delayed during an interrupted treatment due to COVID-19. At present, the COVID-19 pandemic is still prevalent worldwide, and many countries still restrict public activities [21]. This unprecedented situation gives us an unprecedented opportunity to analyze the impact of a sudden interruption of antiVEGF treatment on the stability of patients with nAMD. This study aimed to compare the T\&E and PRN treatment regimens, which are more conducive to the stability of patients with nAMD. Second, we analyzed the changes in the visual acuity and OCT indicators in stable patients with nAMD who had a sudden interruption of anti-VEGF treatment to identify indicators that may predict the disease stability of nAMD, which will be helpful for choosing treatment schemes in the future.

\section{METHODS}

This comparative study of the curative effects using a routine clinical database (single-center retrospective consecutive case series) was carried out in the ophthalmology clinic at the Second Affiliated Hospital of Harbin Medical University, Harbin, China. The study protocol followed the Declaration of Helsinki and was approved by the Institutional Research Board of the hospital (KY2020-261).

\section{Study Population}

Data of the patients with nAMD in our hospital since June 2018 were extracted from the electronic patient records database. The inclusion criteria were as follows: (a) patients with a diagnosis of nAMD that was confirmed by the presence of active leakage of choroidal neovascularization (CNV) detected by fluorescein angiography (FA) or optical coherence tomography angiography (OCTA); (b) patients with nAMD who had treatment before the outbreak of COVID-19 and were followed up at the Ophthalmology Department of the Second Affiliated Hospital of Harbin Medical University during the research period; (c) patients treated with aflibercept $(2 \mathrm{mg} / 0.05 \mathrm{~mL}$, Eylea, Regeneron Pharmaceuticals Inc.) who received a T\&E or PRN therapeutic regimen and who had already reached a stable state; (d) patients with detailed medical records who underwent a complete ophthalmological examination, including the evaluation of at least the best corrected visual acuity (BCVA), intraocular pressure (IOP), slit-lamp examination, optical coherence tomography (OCT), and OCTA. The exclusion criteria were as follows: (a) patients with the presence of CNV related to other etiologies, such as myopia, uveitis, or central serous chorioretinopathy; (b) patients with coexisting ocular disorders, such as retinal vascular occlusion, diabetic retinopathy, retinal vasculitis, or neovascular glaucoma; and (c) patients with incomplete medical records, such as patients who were mainly treated and followed up at other hospitals. Patients whose measurement of OCT and detailed medical records were 
not available, and patients whose BCVA, IOP, OCT, or OCTA data could not be obtained and analyzed for any reason were also excluded.

\section{Study Design}

This study analyzed the patients from the aforementioned database whose treatment schedules were interrupted at least once between 1 February and 31 May 2020 (the period during which the outpatient services and operations were restricted because of the COVID-19 pandemic). The last pre-pandemic visit was recorded as V0, and the first follow-up after the pandemic was recorded as V1. The inclusion of those patients was evaluated independently by two experienced researchers (BJ, LG) on the basis of the aforementioned criteria. Disagreements were adjudicated by a discussion with the senior author (DWS). Cases in which the diagnosis could not be made with certainty were also excluded from this study.

Patients received a strict T\&E protocol. Patients received three consecutive injections every 4 weeks. If a patient was stable after the third visit, the interval was extended by 2 weeks, and the fourth injection was administered after 6 weeks. However, if the patient was unstable, the injection interval was shortened by 2 weeks. The minimum interval was not less than 4 weeks. If a patient's two consecutive follow-up visits with an interval of 12 weeks were stable, it was regarded as the end of treatment (Fig. 2). Stable nAMD was defined as follows: (1) Compared with the previous followup visit, the best-corrected visual acuity (BCVA) deteriorated by no more than five letters. (2) Compared with the last follow-up, the increase in central retinal thickness (CRT) was no more than $10 \%$ on the OCT images. (3) There was no new subretinal fluid (SRF) or/and intraretinal fluid (IRF) on the OCT images. (4) No new hemorrhage or CNV was detected in the OCT or fundic photos. Patients with the PRN therapeutic regimen were followed up once a month after three consecutive injections. On the basis of the results of each monthly examination, the injections were continued if the patient was in an unstable state. For patients with bilateral nAMD requiring treatment, the data of the first eye were selected to be used for the analysis.

The patients had to have the same (true-track based) enhanced depth optical coherence tomography (EDI-OCT) and horizontal single line scan (Heidelberg Engineering, Heidelberg, Germany) encompassing the fovea at each visit. OCT angiography (OCTA, Carl Zeiss Meditec Inc., CA, USA) and fundus fluorescein angiography (FFA, Heidelberg Retina Angiograph 2, Heidelberg Engineering, Heidelberg, Germany) were used to obtain the images. Parameters such as the CRT and the subfoveal choroidal thickness (SFCT) were manually measured by the same doctor (LG). If new $\mathrm{CNV}$ or hemorrhage occurred, then FFA was performed. Demographic characteristics (age, sex, and eye) were recorded. Additionally, the BCVA, CRT, SFCT, type of CNV, OCT features for all visits, number

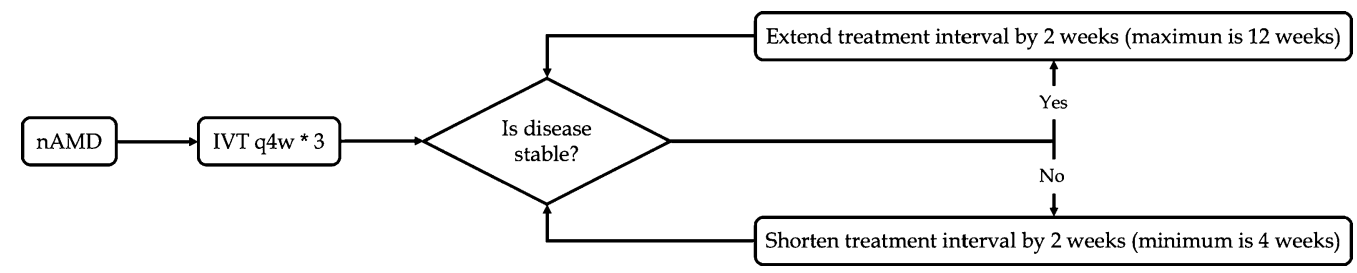

Fig. 2 Outline of the treat and extend regimen for patients with nAMD. Three consecutive injections were administered every 4 weeks; if the patients were stable after the third injection, the interval was extended by 2 weeks, and the fourth injection was administered after 6 weeks. However, if the patients were unstable, the injection interval was shortened by 2 weeks. The minimum interval was never less than 4 weeks. When a patient was stable with a 12 -week treatment interval, two consecutive follow-up visits were conducted to determine the end of treatment 
of injections, and the interval between the last injection and the beginning of the pandemic were evaluated.

\section{Statistical Analysis}

This study was a real-world retrospective analysis. The sample size depended mainly on the number of patients with nAMD who had reached stable status and who had their treatment interrupted during the COVID-19 pandemic. The Snellen BCVA was converted to the Early Treatment Diabetic Retinopathy Study (ETDRS) equivalent for the statistical analysis $[22,23]$. The Shapiro-Wilk method was used to test the normality of the measurement data. The data that were normally distributed are expressed as the mean \pm standard deviation. The $t$ test was used to analyze the difference between the two groups. The measurement data that were not normally distributed were represented by the median (minimum, maximum) and were analyzed using the Mann-Whitney $U$ test. Categorical variables are expressed as $n$ (\%), and the difference between the two groups was analyzed using the chi-square test. A binary logistic regression analysis was used for the univariate analysis to determine the risk factors for patients with stable nAMD, and a $P<0.1$ was used to screen the possible risk factors. All baseline factors were included in the model in the multivariate analysis, and independent variables were screened by the input method. The difference was statistically significant when $P<0.05$. The SPSS 22.0 package program was used for the statistical analysis of the data (IBM, Armonk, New York, USA).

\section{RESULTS}

\section{Demographics and Patient Characteristics}

This study retrospectively analyzed the data of 755 patients with nAMD who received regular treatment with intravitreal injections of aflibercept in June 2018. Of these patients, 209 reached a stable state at the last visit prior to the pandemic outbreak (1 February to 31 May 2020) and after at least 16 weeks of interrupted treatment/follow-up visits (Fig. 3).

The average age of the patients was $69.5 \pm 10.9$ years (92 male, $44.0 \% ; 117$ female, $56.0 \%)$. CNV was type 1 in 98 patients, type 2 in 80 patients, and retinal angiomatous proliferation (RAP) in 31 patients. The CRT was $326.70 \pm 97.57 \mu \mathrm{m}$, and the BCVA was $62.54 \pm 9.72$ letters at the initial treatment. The patient demographics and characteristics are summarized in Table 1 . The patients in the T\&E regimen group were older [analysis of variance (ANOVA), $P=0.01$ ], and there were no significant differences in the other factors between the groups.

\section{Stability of Patients During the COVID-19 Interruption}

With the T\&E regimen, the number of injections given to patients from the initial treatment to VO was greater than with the PRN regimen $(P=0.001)$, and the interval between V0 and the break of the pandemic was shorter $(P=0.031)$. No significant difference was found in the OCT parameters, including the BCVA, CRT, SFCT, fluid in the retina, and pigment epithelial detachment (PED), at Vo between the two groups $(P>0.05$, all) (Table 2$)$.

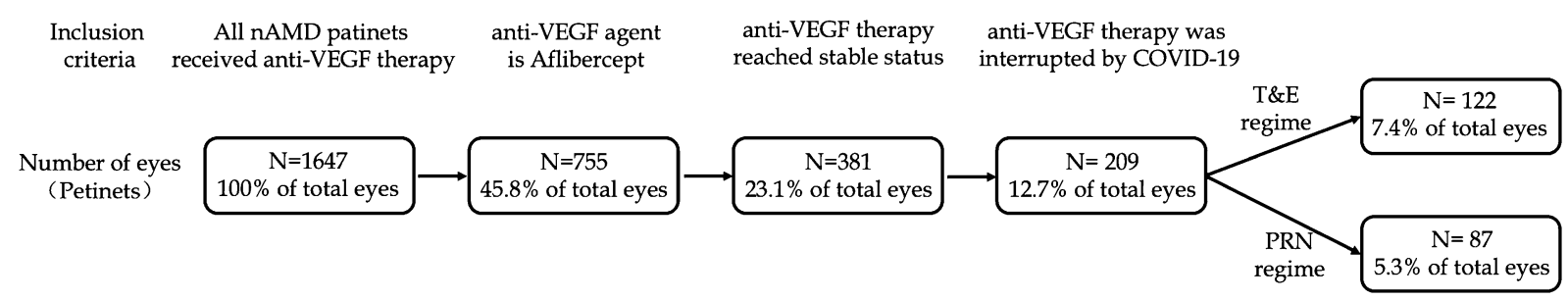

Fig. 3 Consort-style diagram showing the number of eyes in the study and the number and the reason for inclusion 
Table 1 Demographics and characteristics of stable patients with nAMD

\begin{tabular}{llll}
\hline Characteristics & $\begin{array}{l}\text { T\&E regimen } \\
\boldsymbol{n}=\mathbf{1 2 2}\end{array}$ & $\begin{array}{l}\text { PRN regimen } \\
\boldsymbol{n}=\mathbf{8 7}\end{array}$ & $\boldsymbol{P}$ value \\
\hline Age (years) & $72.5(41,90)$ & $65(37,90)$ & 0.001 \\
Male, $n$ (\%) & $51(41.8)$ & $41(47.1)$ & 0.445 \\
Study eye OD, $n$ (\%) & $65(53.3)$ & $39(44.8)$ & 0.228 \\
Initial CNV type & & & \\
Type 1 & $55(45.1)$ & $43(49.4)$ & 0.708 \\
Type 2 & $47(38.5)$ & $33(37.9)$ & \\
RAP & $20(16.4)$ & $11(12.6)$ & \\
Initial BCVA (letters) & $55.32 \pm 9.36$ & $55.26 \pm 9.85$ & 0.967 \\
Initial CRT $(\mu \mathrm{m})$ & $437.57 \pm 135.81$ & $428.14 \pm 115.74$ & 0.600 \\
\hline
\end{tabular}

Data are presented as number (range) or mean \pm SD

$C N V$ choroidal neovascularization, $B C V A$ best-corrected visual acuity, $C R T$ central retinal thickness

Table 2 Characteristics of stable patients with nAMD at last pre-epidemic visit (V0)

\begin{tabular}{llll}
\hline & $\begin{array}{l}\text { T\&E regimen } \\
\boldsymbol{n}=\mathbf{1 2 2}\end{array}$ & $\begin{array}{l}\text { PRN regimen } \\
\boldsymbol{n}=\mathbf{8 7}\end{array}$ & $\boldsymbol{P}$ \\
\hline BCVA (letters) & $62(32-86)$ & $62(32-89)$ & 0.665 \\
CRT $(\mu \mathrm{m})$ & $306(176-533)$ & $322(167-578)$ & 0.107 \\
SFCT $(\mu \mathrm{m})$ & $249.5(173-381)$ & $251(180-358)$ & 0.655 \\
Injection numbers before V0 & $9(4,18)$ & $7(4,15)$ & 0.001 \\
Interval between V0 and the break of pandemic (weeks) & $4(1,11)$ & $5(1,13)$ & 0.031 \\
Presence of fluid (\%) & & & 0.184 \\
Fluid is present & $54(44.3)$ & $48(55.2)$ & $11(12.6)$ \\
$\quad 19(15.6)$ & $19(21.8)$ & \\
$\quad$ Intraretinal fluid & $15(12.3)$ & $18(20.7)$ & \\
$\quad$ Subretinal fluid & $20(16.4)$ & $39(44.8)$ & \\
$\quad$ Both & $68(55.7)$ & $24(27.6)$ & 0.725 \\
No fluid is present & $31(25.4)$ & \\
Presence of PED (\%) & &
\end{tabular}

Data are presented as numbers (proportions) or the range

$B C V A$ best-corrected visual acuity, $C R T$ central retinal thickness, SFCT subfoveal choroidal thickness, PED pigment epithelial detachment

The patients were assessed on the basis of the criteria of stable nAMD. In the T\&E regimen group, patients were more stable than the patients in the PRN regimen group (53.3\% vs. $33.3 \%, P=0.004)$; the BCVA was significantly better than the PRN group (58.5 letters vs. 56 
letters, $P=0.006)$; and the proportion of patients with a BCVA loss of at least five letters was significantly lower at V1 $(27 \%$ vs. $54 \%$, $P<0.001)$. Similarly, the CRT was increased significantly ( $15 \mu \mathrm{m}$ vs. $35 \mu \mathrm{m}, P=0.001)$; and the proportion of patients with an increased CRT over $10 \%$ was higher in the PRN group $(21.3 \%$ vs. $47.1 \%, P<0.001)$. The changes and the average change in the SFCT were greater in the PRN group than in the T\&E group after the COVID-19 interruption $(237 \mu \mathrm{m}$ vs. $257 \mu \mathrm{m}$, $P=0.002 ;-10 \mu \mathrm{m}$ vs. $7 \mu \mathrm{m}, P=0.001)$. In the PRN group, the proportion of patients with an appearance of new fluid was higher at V1
(36.1\% vs. $58.6 \%, P=0.002)$; however, no significant difference was found between the types of fluid, the appearance of new CNV, or the development of hemorrhage between the two groups $(P=0.239, \quad P=0.861, \quad P=0.849)$ (Table 3).

\section{Factors Related to nAMD Disease Stability}

The factors that might affect the stability of the patients with nAMD are summarized in Table 4. Factors that had significant statistical significance during the univariate analysis $(P<0.1)$ and other key factors (including the BCVA,

Table 3 Stability of stable patients with nAMD after COVID-19 interruption

\begin{tabular}{|c|c|c|c|}
\hline & $\begin{array}{l}\text { T\&E regimen } \\
n=122\end{array}$ & $\begin{array}{l}\text { PRN regimen } \\
n=87\end{array}$ & $P$ \\
\hline \multicolumn{4}{|l|}{ V1 status (\%) } \\
\hline Stable & $65(53.3)$ & $29(33.3)$ & \multirow[t]{2}{*}{0.004} \\
\hline Unstable & $57(46.7)$ & $58(66.7)$ & \\
\hline V1 BCVA (letters) & $58.5(31,84)$ & $56(31,86)$ & 0.006 \\
\hline BCVA change from V1 to V0 (letters) & $-3(-20,9)$ & $-5(-17,2)$ & 0.001 \\
\hline BCVA decreased $\geq 5$ letters between V1 to V0 (\%) & $33(27.0)$ & $47(54.0)$ & $<0.001$ \\
\hline V1 CRT $(\mu \mathrm{m})$ & $327(189-713)$ & $379(238-672)$ & 0.001 \\
\hline CRT change between $\mathrm{V} 1$ to $\mathrm{V} 0(\mu \mathrm{m})$ & $15(-188$ to 295$)$ & $35(-20$ to 226$)$ & 0.001 \\
\hline CRT increased $\geq 10 \%$ between V1 to V0 (\%) & $26(21.3)$ & $41(47.1)$ & $<0.001$ \\
\hline V1 SFCT $(\mu \mathrm{m})$ & $237(174-376)$ & $257(187-417)$ & 0.002 \\
\hline SFCT change between $\mathrm{V} 1$ to $\mathrm{V} 0(\mu \mathrm{m})$ & $-10(-95$ to 58$)$ & $7(-52$ to 69$)$ & 0.001 \\
\hline Appearance of new fluid (\%) & $44(36.1)$ & $51(58.6)$ & 0.002 \\
\hline \multicolumn{4}{|l|}{ Type of new fluid (\%) } \\
\hline Intraretinal fluid & $18(14.8)$ & $12(13.8)$ & \multirow[t]{3}{*}{0.239} \\
\hline Both & $33(27)$ & $33(37.9)$ & \\
\hline Subretinal fluid & $71(58.2)$ & $42(48.3)$ & \\
\hline Appearance of new CNV (\%) & $5(4.1)$ & $4(4.6)$ & 0.861 \\
\hline Appearance of new hemorrhages (\%) & $13(10.7)$ & $10(11.5)$ & 0.849 \\
\hline
\end{tabular}

Data are presented as number (proportion) or range

$B C V A$ best-corrected visual acuity, CRT central retinal thickness, SFCT subfoveal choroidal thickness, PED pigment epithelial detachment 
Table 4 Factors related to the stability of patients with nAMD during interruption

\begin{tabular}{|c|c|c|c|c|c|c|}
\hline & \multicolumn{2}{|c|}{$\begin{array}{l}\text { Univariate logistic regression } \\
\text { analysis }\end{array}$} & \multicolumn{2}{|c|}{$\begin{array}{l}\text { Multivariate logistic } \\
\text { regression analysis (plus the } \\
\text { factor of treatment regimen) }\end{array}$} & \multicolumn{2}{|c|}{$\begin{array}{l}\text { Multivariate logistic } \\
\text { regression analysis } \\
\text { (sensitivity analysis plus the } \\
\text { number of injections) }\end{array}$} \\
\hline & OR (95\% CI) & $P$ & OR (95\% CI) & $P$ & OR (95\% CI) & $P$ \\
\hline Age & $1.002(0.976-1.028)$ & 0.903 & & & & \\
\hline Sex (male vs. female) & $0.952(0.55-1.649)$ & 0.862 & & & & \\
\hline Study eye (OD vs. OS) & $1.284(0.743-2.216)$ & 0.37 & & & & \\
\hline \multicolumn{7}{|l|}{ Initial CNV type } \\
\hline Type 2 vs. type 1 & $1.963(1.075-3.585)$ & 0.028 & $2.493(1.179-5.272)$ & 0.017 & $2.500(1.181-5.292)$ & 0.017 \\
\hline RAP vs. type 1 & $2.141(0.928-4.941)$ & 0.074 & $2.912(1.133-7.485)$ & 0.026 & $2.803(1.097-7.167)$ & 0.031 \\
\hline V0 BCVA (letters) & $0.984(0.956-1.012)$ & 0.265 & $1.001(0.967-1.037)$ & 0.937 & $1.004(0.970-1.039)$ & 0.821 \\
\hline V0 CRT $(\mu \mathrm{m})$ & $1(0.997-1.003)$ & 0.931 & $1.000(0.994-1.005)$ & 0.872 & $1.000(0.994-1.005)$ & 0.912 \\
\hline V0 SCT $(\mu \mathrm{m})$ & $1.003(0.995-1.01)$ & 0.477 & $1.002(0.992-1.011)$ & 0.744 & $1.001(0.991-1.010)$ & 0.892 \\
\hline $\begin{array}{l}\text { Injection numbers } \\
\text { before V0 }\end{array}$ & $0.934(0.857-1.018)$ & 0.119 & & & $0.884(0.800-0.977)$ & 0.016 \\
\hline $\begin{array}{l}\text { Interval between V0 } \\
\text { and the break of } \\
\text { epidemic (weeks) }\end{array}$ & $1.394(1.215-1.599)$ & $<0.001$ & $1.371(1.182-1.591)$ & $<0.001$ & $1.438(1.238-1.671)$ & $<0.001$ \\
\hline $\begin{array}{l}\text { Presence of fluid (Y vs. } \\
\mathrm{N} \text { ) }\end{array}$ & $0.990(0.574-1.70)$ & 0.972 & $0.718(0.308-1.671)$ & 0.442 & $0.847(0.361-1.988)$ & 0.703 \\
\hline $\begin{array}{l}\text { Presence of PED (Y vs. } \\
\text { N) }\end{array}$ & $0.556(0.293-1.054)$ & 0.072 & $0.392(0.181-0.852)$ & 0.018 & $0.419(0.195-0.903)$ & 0.026 \\
\hline $\begin{array}{l}\text { Treatment regimen } \\
\text { (T\&E vs. PRN) }\end{array}$ & $0.438(0.248-0.776)$ & 0.005 & $0.437(0.228-0.840)$ & 0.013 & & \\
\hline
\end{tabular}

$C N V$ choroidal neovascularization, $R A P$ retinal angiomatous proliferation, $B C V A$ best-corrected visual acuity, $C R T$ central retinal thickness, SFCT subfoveal choroidal thickness, PED pigment epithelial detachment

CRT, SFCT, and fluid) considered in clinical practice that might affect the stability of nAMD were included in the multivariate logistic regression analysis model. The results showed that stable patients with nAMD with type 1 CNV (OR 2.493 [95\% CI 1.179-5.272], compared with type $2 \mathrm{CNV} ; P=0.017$; OR 2.912 [95\% CI 1.133-7.485], compared with RAP; $P=0.026$ ), with PED (OR $0.392 \quad[95 \% \mathrm{CI}$ 0.181-0.852], compared with absence; $P=0.018$ ) or T\&E regimen (OR 0.437 [95\% CI
0.228-0.840], compared with PRN regimen; $P=0.013)$ were more likely to remain stable when treatment was interrupted.

The injection numbers and treatment regimens were not mutually independent factors due to the interruption caused by the outbreaks. Therefore, a sensitivity analysis of the frequency of injection at V0 (multivariate analysis with the aforementioned factors) was conducted. The results showed that the risk of disease instability could be reduced by $11.6 \%$ for each 
additional injection before the interruption of the pandemic. Compared with type $1 \mathrm{CNV}$, type $2 \mathrm{CNV}$ increased the risk of disease instability by at least $17.9 \%$, and RAP increased the risk of disease instability by at least $13.3 \%$. The risk of disease instability increased by $37.1 \%$ with the increase in the interval between the last injection (V0) and the beginning of the pandemic. Compared with patients without PED, patients with PED at the last injection had a $61.8 \%$ lower risk of disease instability on average. Compared with the PRN group, the risk of disease instability in the T\&E group decreased by $56.3 \%$.

\section{DISCUSSION}

The effect of anti-VEGF therapy on patients with nAMD has been widely recognized [4-6]. However, patients need regular visits and a long treatment regime, which in reality increases the difficulty of treatment [8,9]. The types of treatment schemes that easily achieve a stable effect and the kinds of patients that can have treatment stopped have been research hotspots in recent years [24, 25]. The COVID-19 pandemic gives us such an unexpected opportunity to observe the stability of patients under the condition of interrupted treatment. This study assessed the status of stable patients with nAMD before and after treatment interruption and found that patients with the T\&E regimen were more resistant to any sudden interruptions of treatment, with the CRT relative fluctuation and the deterioration of vision. We are also trying to explore the factors that may help to identify or maintain stability in patients with such treatment interruptions. Patients with type $1 \mathrm{CNV}$ or patients with PED were more likely to remain stable when treatment was interrupted.

Compared with the monthly continuous treatment plan and the PRN regimen, the T\&E regimen is a positive treatment regimen because it changes the injection interval. It avoids individuation, over- or undertreatment, and the activation of recurrent diseases, fluid accumulation, and irreversible tissue damage caused by the PRN treatment [12-15]. In some published real-world studies, the T\&E protocol still demonstrated the effectiveness of treatment and the stability caused by the treatment $[26,27]$. However, patients with nAMD who need long-term follow-up and treatment, especially elderly patients, are more likely to stop follow-up visits or treatment for any reason, such as when these patients develop sudden physical dysfunction or family issues. In this study, patients with stable nAMD who received the T\&E treatment regimen were still in a stable state $(55.3 \%)$, although the situation of the pandemic suddenly interrupted treatment longer than the maximum treatment extension time (16 weeks), and approximately $27 \%$ of the patients had their vision decreased by more than five letters. Although 33.3\% of the patients treated with the PRN regimen were stable after treatment interruption, approximately $54 \%$ of the patients had significantly decreased vision by more than five letters (Fig. 4). A retrospective analysis by Nguyen et al. found that $41 \%$ of eyes showed reactivation within the first year after treatment suspension, which increased to $79 \%$ at 5 years [28]. Essex et al. presented a detailed analysis of the observed treatment patterns and visual results during the maintenance phase of the treat and extend regimens for nAMD in a real-world cohort of patients. The risk of reactivation increased when the treatment interval was increased, and the risk of reactivation increased substantially when the treatment interval extended beyond 12 weeks and reached $36.5 \%$ if the interval was 20 weeks or more from the previous injection [29]. Even after completing the T\&E protocol, $29.4 \%$ of patients showed a recurrence of CNV [30].

Despite a relatively high frequency of $T \& E$ injections (9 times on average, 4-18 times), the results of this study were consistent with the results of many studies because of the treatment scheme itself [31]. Although the number of injections was greater, we found that every additional injection before the sudden interruption could reduce the risk of disease instability by $11.6 \%$. Some studies have pointed out that it is necessary to avoid an early termination of treatment in order to avoid recurrence of the disease because the vision gained during the course of therapy could potentially be lost if the 


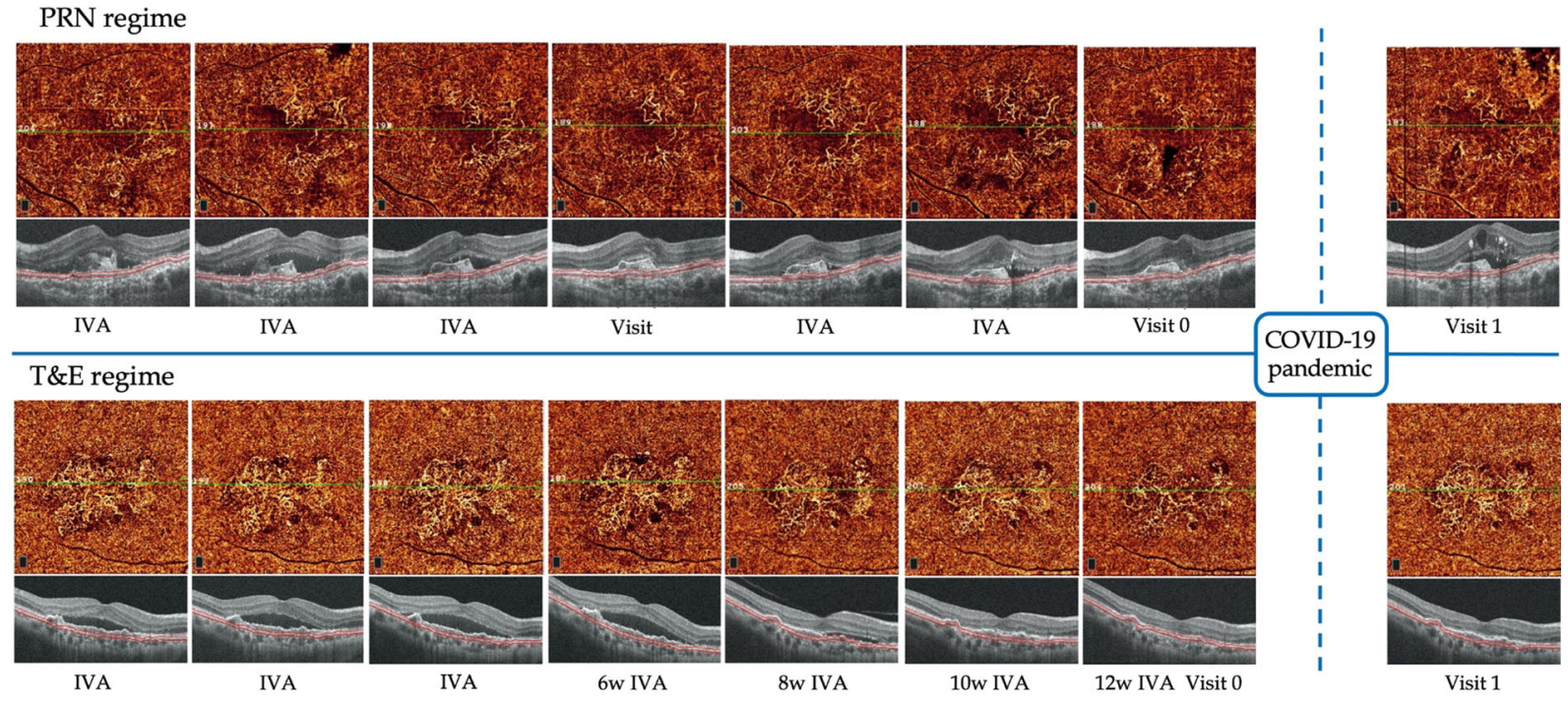

Fig. 4 OCTA images of the patients with nAMD treated with the T\&E and PRN regimes interrupted by the COVID-19 pandemic. The top line is the OCTA images of a 65-year-old patient with nAMD who received the PRN regime; after three consecutive injections every 4 weeks, there was no injection in the fourth follow-up, but at the fifth follow-up, there was subretinal fluid (SRF) recurrence, and the patient received an injection. During the sixth follow-up period, the fluid was still not absorbed, and the patient received an injection. It was stable at the

eyes develop a reactivation of the disease, and the improvements made during treatment may not be regained even if treatment is resumed [30]. For patients with the T\&E regimen, it was not necessary to undergo follow-up every month until the occurrence of instability, the number of times they needed to come to the hospital was less, and the timing of the next treatment was predictable. During the outbreak of COVID-19, some guidelines were issued to help guide ophthalmologists in managing patients with anti-VEGF therapy [17, 32, 33]. However, these guidelines are limited to the protection of specific procedures such as outpatient and surgical procedures, but few guidelines are available to select treatment regimens. Therefore, in the case of major events or emergencies, patients with the T\&E regimen can better plan their visits to the hospital, which can reduce the risk of exposure to COVID-19. last follow-up (V0); however, after the outbreak, the lesion became unstable again, and subretinal fluid and intraretinal fluid (IRF) were observed on OCT images. The bottom line is the OCTA images of a 73-year-old patient with $\mathrm{nAMD}$ who received the T\&E regime; this patient received three consecutive injections every 4 weeks; the patient was stable, and the interval was extended by 2 weeks at each visit; the seventh injection interval was extended 12 weeks once (V0). After the outbreak, this patient was still stable

Many studies have reported that in the initial treatment, some factors, such as age, visual acuity, the CNV type, and CRT, could predict the prognosis of patients with anti-VEGF treatment [34-36]. In this study, we found that SFCT was associated with significant reductions in the T\&E regimen compared with the PRN regimen. Choroidal circulation is essential to maintain normal visual function by providing nutrients and removing metabolic wastes from the outer retina and the retinal pigmented epithelium. The choroid plays an important role in the pathophysiology of common chorioretinal diseases such as AMD [3]. Choroidal thickening may, therefore, occur in the active phase of nAMD because of the increased levels of VEGF and other proinflammatory factors together with hydrostatic variation in the blood flow within the newly formed vessels [37]. Invernizzi et al. found significant choroidal thickening in all of the CNV types [38]. Koizumi et al., Kaya, 
and Mazaraki et al. evaluated the changes in the choroidal thickness following intravitreal injections. They showed that treatment-naive and pretreated eyes had significant reductions in the choroidal thickness in all locations [39-41]. At present, the analysis was lacking with regard to the factors that were more likely to obtain a stable treatment effect in patients during the process of treatment. In this study, we also analyzed the factors related to the stability of the patients whose treatment had already reached a stable state even when the planned treatment was not implemented because of the impact of the pandemic and when treatment was interrupted for at least 16 weeks. Patients receiving the T\&E regimen had a $56.3 \%$ reduced risk of disease instability compared with those patients receiving the PRN regimen. We found that among the types of $\mathrm{CNV}$ at the time of initial treatment, the treatment of patients with type $1 \mathrm{CNV}$ could easily achieve stability. Nakano et al. found that type $1 \mathrm{CNVs}$ show significantly lower vessel junction densities than type $2 \mathrm{CNVs}$ on OCTA, suggesting that type $1 \mathrm{CNVs}$ have more mature vessels and that type $1 \mathrm{CNVs}$ can persist in a quiescent, noninfiltrative, trophic state below the RPE after treatment [42]. Patients with PED were also more likely to remain stable when treatment was interrupted. Recently, Christenbury et al. found that macular atrophy progresses significantly slower in areas corresponding to PED containing neovascular type 1 CNV flow on OCTA [43], and the presence of PED may protect the macular area from "burnt out" [44]. Therefore, treatment in patients with these characteristics could result in a better stability, patients can maintain the stability for a longer time, and the treatment intervals could be gradually extended when there are sudden interruptions. At present, no standard protocol exists for when to stop antiVEGF treatment in patients with nAMD. Therefore, we can conclude that patients do not easily relapse at the end of treatment or after an interruption, and this study is also an exploration of end-of-treatment indicators.

This study had some limitations. First, the results may not be extrapolated to other populations because this was a single-center study with a relatively small sample size. Second, this was a retrospective study. Because of the many confounding factors, some bias might exist in the results. However, randomized controlled studies do not have the opportunity to investigate a pool of patient data from patients who have a sudden interruption of treatment. Therefore, this study might have significance during some exceptional situations, such as during the COVID-19 pandemic. Finally, the clinical application of aflibercept in patients with nAMD receiving the T\&E regimen has been approved in China since 2018. To date, no realworld evidence comparing the T\&E and PRN regimens to evaluate the effect of disease stability with the use of aflibercept in patients with nAMD has been conducted in China. Realworld studies with long-term follow-up to evaluate the disease stability between the different regimens still need to be conducted. Therefore, anti-VEGF therapy in the real world in China will be the direction of research in the future.

\section{CONCLUSIONS}

Compared to patients receiving the PRN treatment regimen, the stable patients with nAMD receiving the T\&E treatment regimen could maintain better stability when their treatments were suddenly interrupted by the COVID-19 pandemic. In addition, patients with type 1 CNV or patients with PED are more likely to remain stable. At present, the COVID-19 pandemic is becoming increasingly normalized, and the T\&E regimen can become a more advanced treatment option for patients in therapy, because the T\&E regimen can not only reduce the number of follow-ups and avoid hospital infection but also effectively maintain the level of anti-VEGF, reduce reactivation of their lesions, and maintain stable vision.

\section{ACKNOWLEDGEMENTS}

Funding. This study was supported by the National Natural Science Foundation of China 
(No. 82171103). The journal's Rapid Service and Open Access fees were funded by the authors.

Editorial Assistance. A professional English proofreading company (AJE) revised this manuscript.

Authorship. All named authors meet the International Committee of Medical Journal Editors (ICMJE) criteria for this article, take responsibility for the integrity of the work as a whole, and have given their approval for this version to be published.

Author Contributions. Sun DW designed the study and adjudicated the study; Jiang B performed the majority of the study and wrote the manuscript; Lin G performed the majority of the study and analyzed the data; all other authors contributed equally to the collection and analysis of the data; and all authors read and approved the final manuscript.

Disclosures. Bo Jiang, Lin Gao, Su Dong, Qingxue Hou, Minghao Sun, Jingjie Zhang, Haotian Yu, Zhongyu Zhang and Dawei Sun have nothing to disclose.

Compliance with Ethics Guidelines. The study was approved by the institutional review board (IRB), the Human Research Ethics Committee of the Second Affiliated Hospital of Harbin Medical University (KY2020-261). It fully complied with the ethical standards of the hospital and followed the Helsinki Declaration of 1964 and its later amendments. All of the participants who provided consent for publication-identified information are included in the manuscript. All participants agreed to publish the information contained in the manuscript.

Data Availability. The data generated during and/or analyzed during the current study are available from the corresponding author on reasonable request.

Open Access. This article is licensed under a Creative Commons Attribution-NonCommercial 4.0 International License, which permits any non-commercial use, sharing, adaptation, distribution and reproduction in any medium or format, as long as you give appropriate credit to the original author(s) and the source, provide a link to the Creative Commons licence, and indicate if changes were made. The images or other third party material in this article are included in the article's Creative Commons licence, unless indicated otherwise in a credit line to the material. If material is not included in the article's Creative Commons licence and your intended use is not permitted by statutory regulation or exceeds the permitted use, you will need to obtain permission directly from the copyright holder. To view a copy of this licence, visit http://creativecommons.org/licenses/by$\mathrm{nc} / 4.0 /$.

\section{REFERENCES}

1. Kim JH, Chang YS, Kim JW. Natural course of patients discontinuing treatment for age-related macular degeneration and factors associated with visual prognosis. Retina. 2017;37(12):2254-61.

2. Wong TY, Chakravarthy U, Klein R, et al. The natural history and prognosis of neovascular age-related macular degeneration: a systematic review of the literature and meta-analysis. Ophthalmology. 2008;115(1):116-26.

3. Wong WL, Su X, Li X, et al. Global prevalence of age-related macular degeneration and disease burden projection for 2020 and 2040: a systematic review and meta-analysis. Lancet Glob Health. 2014;2(2):e106-16.

4. Rosenfeld PJ, Brown DM, Heier JS, et al. Ranibizumab for neovascular age-related macular degeneration. N Engl J Med. 2006;355(14):1419-31.

5. Brown DM, Michels M, Kaiser PK, Heier JS, Sy JP, Ianchulev T. Ranibizumab versus verteporfin photodynamic therapy for neovascular age-related macular degeneration: two-year results of the ANCHOR study. Ophthalmology. 2009;116(1):5765.e5.

6. Solomon SD, Lindsley K, Vedula SS, Krzystolik MG, Hawkins BS. Anti-vascular endothelial growth factor for neovascular age-related macular degeneration. Cochrane Database Syst Rev. 2014;8(8): CD005139. 
7. Sarwar S, Clearfield E, Soliman MK, et al. Aflibercept for neovascular age-related macular degeneration. Cochrane Database Syst Rev. 2016;2:CD011346.

8. Rofagha S, Bhisitkul RB, Boyer DS, Sadda SR, Zhang K. Seven-year outcomes in ranibizumab-treated patients in ANCHOR, MARINA, and HORIZON: a multicenter cohort study (SEVEN-UP). Ophthalmology. 2013;120(11):2292-9.

9. Schmidt-Erfurth U, Kaiser PK, Korobelnik JF, et al. Intravitreal aflibercept injection for neovascular age-related macular degeneration: ninety-six-week results of the VIEW studies. Ophthalmology. 2014;121(1):193-201.

10. Lalwani GA, Rosenfeld PJ, Fung AE, et al. A variabledosing regimen with intravitreal ranibizumab for neovascular age-related macular degeneration: year 2 of the PrONTO Study. Am J Ophthalmol. 2009;148(1):43-58.e1.

11. Wecker T, Grundel B, Reichl S, et al. Anti-VEGF injection frequency correlates with visual acuity outcomes in pro re nata neovascular AMD treatment. Sci Rep. 2019;9(1):3301.

12. Amoaku $\mathrm{W}$, Balaskas $\mathrm{K}$, Cudrnak $\mathrm{T}$, et al. Initiation and maintenance of a treat-and-extend regimen for ranibizumab therapy in wet age-related macular degeneration: recommendations from the UK Retinal Outcomes Group. Clin Ophthalmol. 2018;12: 1731-40.

13. DeCroos FC, Reed D, Adam MK, et al. Ttreat-andextend therapy using aflibercept for neovascular age-related macular degeneration: a prospective clinical trial. Am J Ophthalmol. 2017;180:142-50.

14. Traine PG, Pfister IB, Zandi S, Spindler J, Garweg JG. Long-term outcome of intravitreal aflibercept treatment for neovascular age-related macular degeneration using a "treat-and-extend" regimen. Ophthalmol Retina. 2019;3(5):393-9.

15. Jaki Mekjavic P, Zaletel BP. Outcome of 5-year treatment of neovascular age-related macular degeneration with intravitreal anti-vegf using "treat and extend" regimen. Front Med (Lausanne). 2018;5:125.

16. Ishibashi K, Haruta M, Ishibashi $Y$, Noda R, Dake S, Yoshida S. Four-year outcomes of intravitreal aflibercept treatment for neovascular age-related macular degeneration using a treat-and-extend regimen in Japanese patients. Ther Adv Ophthalmol. 2021;13:2515841420984586.

17. Wong TY, Bandello F. Academic ophthalmology during and after the COVID-19 pandemic. Ophthalmology. 2020;127(8):e51-51e52.
18. American Academy of Ophthalmology. Recommendations for urgent and nonurgent patient care. $2020 . \quad$ https://www.aao.org/headline/ recommendations-urgent-nonurgent-patient-care. Accessed 18 Mar 2020.

19. Yang KB, Feng H, Zhang H. Effects of the COVID-19 pandemic on anti-vascular endothelial growth factor treatment in China. Front Med (Lausanne). 2020;7:576275.

20. Zhao X, Meng L, Luo M, et al. The influence of delayed treatment due to COVID-19 on patients with neovascular age-related macular degeneration and polypoidal choroidal vasculopathy. Ther Adv Chronic Dis. 2021;12:20406223211026388.

21. Mack HG, Fraser-Bell S. "COVID new normal" in ophthalmology: Implications for ophthalmologists, eye care, ophthalmic education and research. Clin Exp Ophthalmol. 2021;49(1):9-11.

22. Kaiser PK. Prospective evaluation of visual acuity assessment: a comparison of snellen versus ETDRS charts in clinical practice (An AOS Thesis). Trans Am Ophthalmol Soc. 2009;107:311-24.

23. Falkenstein IA, Cochran DE, Azen SP, et al. Comparison of visual acuity in macular degeneration patients measured with Snellen and Early Treatment Diabetic Retinopathy Study charts. Ophthalmology. 2008;115(2):319-23.

24. Menke MN, Zinkernagel MS, Ebneter A, Wolf S. Functional and anatomical outcome of eyes with neovascular age-related macular degeneration treated with intravitreal ranibizumab following an exit strategy regimen. Br J Ophthalmol. 2014;98(9): 1197-200.

25. Arendt $\mathrm{P}, \mathrm{Yu} \mathrm{S}$, Munk MR, Ebneter A, Wolf $\mathrm{S}$, Zinkernagel MS. Exit strategy in a treat-and-extend regimen for exudative age-related macular degeneration. Retina. 2019;39(1):27-33.

26. Daien V, Finger RP, Talks JS, et al. Evolution of treatment paradigms in neovascular age-related macular degeneration: a review of real-world evidence. Br J Ophthalmol 2020;105(11):1475-9.

27. Carrasco J, Pietsch GA, Nicolas MP, Koerber C, Bennison C, Yoon J. Real-world effectiveness and real-world cost-effectiveness of intravitreal aflibercept and intravitreal ranibizumab in neovascular age-related macular degeneration: systematic review and meta-analysis of real-world studies. Adv Ther. 2020;37(1):300-15.

28. Nguyen V, Vaze A, Fraser-Bell S, et al. Outcomes of suspending VEGF inhibitors for neovascular agerelated macular degeneration when lesions have 
been inactive for 3 months. Ophthalmol Retina. 2019;3(8):623-8.

29. Essex RW, Nguyen V, Walton R, et al. Treatment patterns and visual outcomes during the maintenance phase of treat-and-extend therapy for agerelated macular degeneration. Ophthalmology. 2016;123(11):2393-400.

30. Adrean SD, Chaili S, Grant S, Pirouz A. Recurrence rate of choroidal neovascularization in neovascular age-related macular degeneration managed with a treat-extend-stop protocol. Ophthalmol Retina. 2018;2(3):225-30.

31. Adrean SD, Chaili S, Ramkumar H, Pirouz A, Grant S. Consistent long-term therapy of neovascular agerelated macular degeneration managed by 50 or more anti-VEGF injections using a treat-extendstop protocol. Ophthalmology. 2018;125(7): 1047-53.

32. Korobelnik JF, Loewenstein A, Eldem B, et al. Guidance for anti-VEGF intravitreal injections during the COVID-19 pandemic. Graefes Arch Clin Exp Ophthalmol. 2020;258(6):1149-56.

33. Corazza P, D'Alterio FM, Younis S. Proposed algorithm during COVID-19 pandemic for patient management in medical retina clinic. Int J Retina Vitreous. 2020;6:20.

34. Chakravarthy U, Pillai N, Syntosi A, Barclay L, Best C, Sagkriotis A. Association between visual acuity, lesion activity markers and retreatment decisions in neovascular age-related macular degeneration. Eye (Lond). 2020;34(12):2249-56.

35. Lai TT, Hsieh YT, Yang CM, Ho TC, Yang CH. Biomarkers of optical coherence tomography in evaluating the treatment outcomes of neovascular age-related macular degeneration: a real-world study. Sci Rep. 2019;9(1):529.

36. Zhang X, Lai T. Baseline predictors of visual acuity outcome in patients with wet age-related macular degeneration. Biomed Res Int. 2018;2018:9640131.
37. Bhisitkul RB. Vascular endothelial growth factor biology: clinical implications for ocular treatments. Br J Ophthalmol. 2006;90(12):1542-7.

38. Invernizzi A, Benatti $\mathrm{E}$, Cozzi $\mathrm{M}$, et al. Choroidal structural changes correlate with neovascular activity in neovascular age related macular degeneration. Invest Ophthalmol Vis Sci. 2018;59(10): 3836-41.

39. Koizumi H, Kano M, Yamamoto A, et al. Subfoveal choroidal thickness during aflibercept therapy for neovascular age-related macular degeneration: twelve-month results. Ophthalmology. 2016;123(3):617-24.

40. Kaya F. Change in choroidal thickness after intravitreal injection for treatment of neovascular age-related macular degeneration: ranibizumab versus aflibercept. J Fr Ophtalmol. 2017;40(10): 832-8.

41. Mazaraki K, Fassnacht-Riederle H, Blum R, Becker $\mathrm{M}$, Michels S. Change in choroidal thickness after intravitreal aflibercept in pretreated and treatmentnaive eyes for neovascular age-related macular degeneration. $\mathrm{Br} \mathrm{J}$ Ophthalmol. 2015;99(10): 1341-4.

42. Nakano Y, Kataoka K, Takeuchi J, et al. Vascular maturity of type 1 and type 2 choroidal neovascularization evaluated by optical coherence tomography angiography. PLoS ONE. 2019;14(4):e0216304.

43. Christenbury JG, Phasukkijwatana N, Gilani F, Freund KB, Sadda S, Sarraf D. Progression of macular atrophy in eyes with type 1 neovascularization and age-related macular degeneration receiving longterm intravitreal anti-vascular endothelial growth factor therapy: an optical coherence tomographic angiography analysis. Retina. 2018;38(7):1276-88.

44. Dansingani KK, Freund KB. Optical coherence tomography angiography reveals mature, tangled vascular networks in eyes with neovascular age-related macular degeneration showing resistance to geographic atrophy. Ophthalmic Surg Lasers Imaging Retina. 2015;46(9):907-12. 